PoS $\quad \begin{aligned} & \text { PROCEEDINGS } \\ & \text { OF SCIENCE }\end{aligned}$

\title{
Experimental particle physics in the LHC era and possible implications for development in Africa
}

\author{
Kétévi Adiklè Assamagan*† \\ Brookhaven National Laboratory \\ E-mail: ketevi@bnl.gov
}

A review of the participation of African institutes in experimental particle physics research in the LHC area is presented. Implications for development and capacity building in Africa are discussed.

Kruger 2010: Workshop on Discovery Physics at the LHC

December 5 - 10, 2010

Kruger National Park, Mpumalanga, South Africa

\footnotetext{
* Speaker.

$\dagger$ On the behalf of Organising Committee of the First African School of Fundamental Physics in Stellenbosch, South Africa, 1-21 August 2010
} 


\section{Introduction}

A school of fundamental physics and its applications, ASP2010, took place in Stellenbosch, South Africa, on August 1-21, 2010 [1]. The school was based on a close interplay between theoretical, experimental, and applied physics. It covered a wide range of topics: particle physics, particle detectors, astro-particle physics and cosmology, computing, accelerator technologies and some of the applications, such as medical physics, light sources and magnetic confinement fusion. The participating students were selected from all over Africa and beyond. A selection of lecture topics in theory, experimental and applied physics was proposed for the school. Scientists from Africa, Europe and the USA were invited to prepare and deliver lectures according to the proposed topics taking into account the diverse levels of the students. The duration of the school allowed for networking - interactions among students and between students and lecturers. The school was funded by institutes in Africa, Europe and the USA.

The motivation to carry out such a school in Africa is presented in Section 2. The organisation of the school is discussed in Section 3, this includes a careful selection of the venue, of the curriculum, the financial support for the school and the selection of the students. In Section 4, we discuss the school itself, i.e., the activities during the period August 1-21, 2010 when the school took place in Stellenbosch: The logistical support offered by the host country and how this contributed to the success of the school, the lecture material that was presented to the students, and finally the discussion and practical sessions that were organised to reinforce the understanding of the lectures and to promote networking. In Section 5, we present the activities after the school, which included balancing the budget, the obtaining of feedback from and maintaining contacts with the students. In Section 6, we discuss the prospects of organising the school again in 2012. And finally, some concluding remarks are offered in Section 7.

\section{Motivation}

The aim of the school is to contribute to capacity building in Africa by harvesting, interpreting, and exploiting the results of current and future physics experiments with particle accelerators, and increasing proficiency in related applications and technologies. As an example, we discuss the opportunities offered by the Large Hadron Collider (LHC) [2] and its experiments, although the basic objective is to help improve the quality of higher education in Africa, to help increase the number of African students acquiring higher education. We believe that the knowledge students gain will benefit them in whichever careers they may pursue. Many students trained in fundamental physics go into industry, become educators or go into basic research.

Large experiments at the LHC and the Tevatron [3] have drawn on the involvement of research institutes and universities from around the world. Some of the salient LHC experiments include ATLAS, CMS, ALICE and LHCb [4, 5, 6, 7]. ATLAS and CMS are general purpose experiments (for precision tests of the Standard Model of elementary particles and the search for new physics beyond the Standard Model) whereas ALICE and LHCb are dedicated experiments to study the early universe and the matter-antimatter asymmetry in the universe. These are very complex and intricate detectors whose design and operation necessitate the collaboration of many physicists and engineers from around the world. The ATLAS collaboration for example consists of upwards of 
3000 physicists spread across different countries and time zones. The LHC itself is the highest energy accelerator ever built; it has a circumference of $27 \mathrm{~km}$ and accelerates and collides proton bunches to $7 \mathrm{TeV}$. Heavy ion collisions have started in November 2010. These collisions are expected to re-create some of the conditions that existed in the early universe or to create elementary particles whose existence would enhance our understanding of the dynamics between matter and forces in the universe. Indeed some of the questions that could be answered at the LHC by ATLAS, CMS, ALICE and LHCb experiments include the nature of Dark Matter, the electroweak symmetry breaking and the generation of mass for elementary particles, the origin of matter-antimatter asymmetry in the universe, the state of the quark-gluon plasma. We therefore have a cutting edge accelerator equipped with very complex detectors built with a variety of detection techniques. The operation of these detectors requires efficient triggering system to sift through the very high rate of the LHC collisions and identify the interesting events whose detailed analysis could shed some light on the aforementioned fundamental questions of particle physics, astro-particle physics and cosmology. The complete system of the LHC and its detectors, together with efficient triggering mechanisms to select interesting events, is complemented by a network of data sharing based on the Grid. Indeed, within less than a few days of recording the data at CERN, these are distributed through the Grid to many centres around the world, allowing different research groups to partake in the analyses without explicit presence at CERN.

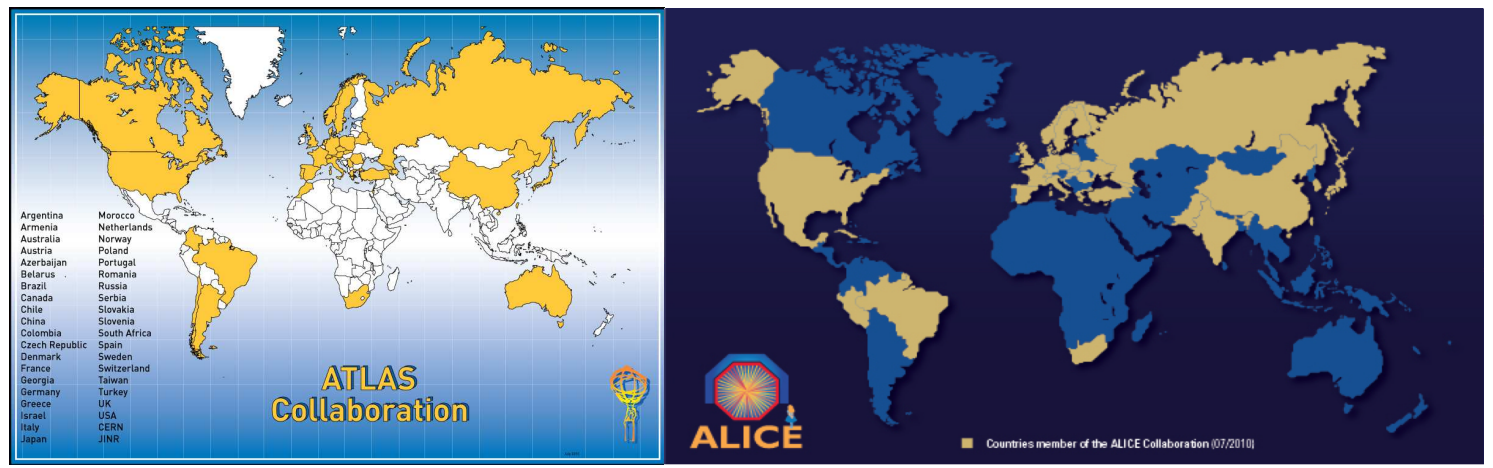

Figure 1: The distribution of participating countries in the ATLAS (left) and ALICE (right) projects. From Africa, only Morocco and South Africa are involved in ATLAS. South Africa is the only African country in ALICE.

These LHC projects are world wide efforts and the African participation and contributions can still be increased. For example, the only African countries in the ATLAS project are Morocco and South Africa as shown in Fig. 1 (left). Only South Africa is in the ALICE project with two institutes, namely the University of Cape Town and iThemba LABS, see Fig. 1 (right). Egypt and Tunisia are the only African countries in the CMS experiment. At the time of writing, there are no African universities or research institutes in the LHCb collaboration. Figure 2 shows the distribution of CERN users from various countries where it is evident that the participation of African scientists is not significant. These LHC projects offer significant capacity building for the countries involved. For example, on the ATLAS experiment alone, there are about 900 Ph.D. students, similarly for the CMS experiment. Furthermore, undergraduate students participate in summer student programs at CERN where they are offered the opportunity to work with experimentalists and 


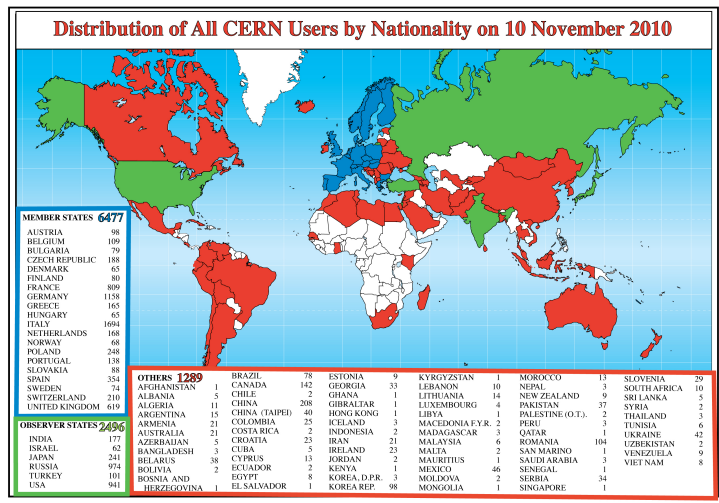

Figure 2: A distribution of CERN users per country. The fraction of CERN users from African countries is about $0.5 \%$.

theorists on various projects. The fraction of Ph.D. students from Africa on these experiments or the fraction of undergraduate students from Africa participating in the summer student programs at CERN is nearly negligible.

We have taken the example of projects at the LHC to motivate the needs to increase capacity building in basic science in Africa. The situation presented above is not limited to projects at CERN but reflects the general trend in major research facilities around the world.

The primary motivation for ASP2010 is to increase the knowledge and improve the capacity building of African students. We expect to achieve this through an outreach effort, an increased awareness of the potential of high quality training offered within the LHC context in various disciplines not just particle physics, and a system of networking. ASP2010 is a positive step towards the broader objectives of the school. We hope to continue with another school in 2012, ASP2012, and in doing so, help increase the global presence of African students and scientists.

\section{Organisation of the School}

In this section, we discuss the organisation of ASP2010, i.e., all the preparatory activities necessary to ensure the success of the school. The preparation for ASP2010 took some time, from its conception to realisation. Late in 2008, there was a firm commitment from Centre National de la Recherche Scientifique (CNRS)/IN2P3 in France to support and fund this project. This was the encouragement needed to seek the additional financial support required to cover the total budget for the school, as discussed in Section 3.3. The first milestone was achieved with a proposal for a school in Africa submitted to the ICTP [8] in February 2009. It was the beginning of concerted efforts on ASP2010.

\subsection{Selection of the Venue}

The selection of the host country was very important because the support offered by the host country has a large impact on the success of the school. Since ASP2010 was primarily targeted 
towards African countries below the Sahara, the host country was considered from that region. A few options were explored in West Africa, Central Africa and Southern Africa. After several considerations, South Africa was finally selected as the host for ASP2010. Some of the considerations that went into this decision include: the logistical infrastructure that is required for the school, and the ability of the host country to provide such a support; the ability to put together a local organising committee dedicated to the objective and the success of ASP2010, and directly involved in the preparation of the school; the prior experience - that may have been accumulated in the host country - from previous schools held in the country in question; the existence of physics teaching capacity in local universities up to at least the Bachelor degree; the existence of some local research/teaching in fundamental physics; the support from the local government in terms of the concrete financial contribution towards the running of the school.

After identifying South Africa as the host for ASP2010, the venue of the school within South Africa was then discussed. Many viable options were explored, taking into account the timing of the school and some of the considerations mentioned above. Ultimately, the National Institute of Theoretical Physics, NITheP [9] in Stellenbosch, was selected as the venue. Subsequently, members of the organising committee made a couple of visits to South Africa to see the selected location and the infrastructure that would be available for lectures, discussion and practical sessions, for hosting the students and the lecturers. In Section 4.1, we discuss how the logistical support contributed to the success of the school. It is expected that the next school, ASP2012, will be hosted by another Sub-Saharan African country and the same criteria outlined above to select the host will apply.

\subsection{Courses}

Four main topics formed the backbone of the school: Theoretical Fundamental Physics, Experimental Subatomic Physics, Information Technology and Grid computing, and Accelerators and Applied Technologies.

Each topic is further divided into an initial set of recaps of essential background knowledge, followed by four main lecture themes, and finally a dedicated theme on computing-related aspects of the topic, including Monte Carlo generators, Grid computing, and high-performance computing. The latter was structured partly into hands-on practical sessions. Further, each main topic contained a number of additional exercises. These were completed in groups, with a single lecturer (mentor) assigned to each group. These groups also provided opportunities for discussing questions arising from the lecture material. The groups were assigned on arrival, and time was reserved for this activity each working day during the school. These daily discussion sessions provided a framework for mentoring students from different backgrounds. Finally, special ${ }^{1}$ lectures were organised during the school, to highlight the edge of current research and topics of special interest to the host region. These were more pedagogical in nature, and were open to a wider audience, e.g., from the host institution and its surroundings. There was one such talk for each of the three main scientific themes.

\footnotetext{
${ }^{1}$ These were motivational speeches, see "star" lectures on the agenda [1].
} 


\subsection{Financial Support}

The school was sponsored by an unprecedented large number of international physics institutes and organisations. These included Spain: AECID Ministry of Foreign Affairs, France: Centre National de la Recherche Scientifique (CNRS)-IN2P3, Institut des Grilles and Commissariat à Ãğl'énergie atomique (CEA), Switzerland: Ecole Polytechnique Fédérale de Lausanne (EPFL) and Paul Scherrer Institute (PSI), South Africa: NITheP and National Research Foundation (NRF), and the USA: Fermilab (FNAL), Department of Energy (DOE), Brookhaven National Laboratory (BNL), Jefferson Lab, National Science Foundation (NSF), CERN and ICTP. In addition, the International Union of Pure and Applied Physics (IUPAP) offered travel grants to five female students.

We managed to collect a total budget of $€ 157,070$ as shown in Table 1 . Funds provided by local institutions, namely the NITheP and the NRF, were spent exclusively to cover local expenses.

Travel expenses of some lecturers were covered by their home institutes, in addition to the contributions listed in Table 1. This was a crucial support for ASP2010 and represents 55\% of lecturers travel budget amounting to $€ 40,000$ as illustrated in Fig. 3.

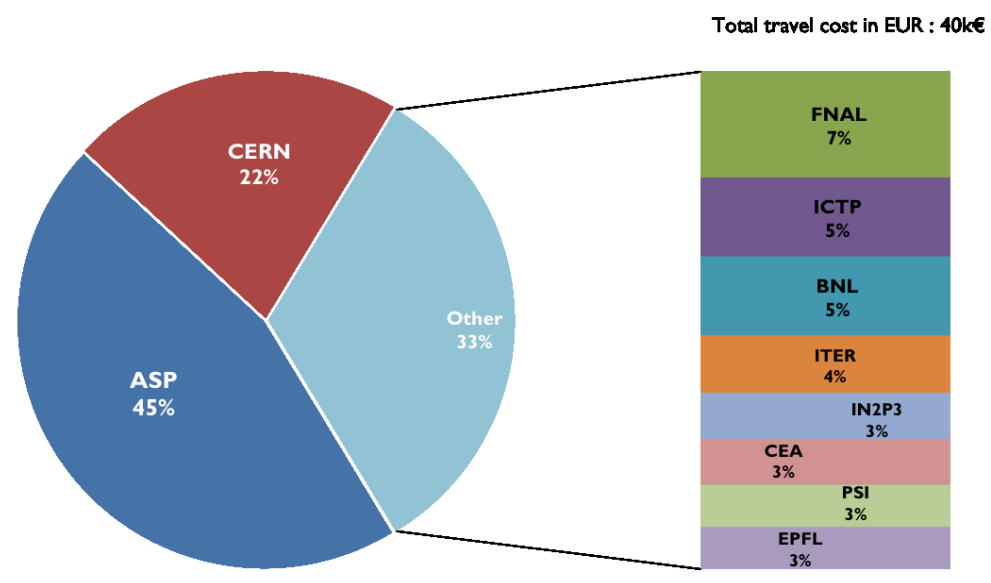

Figure 3: Travel expenses covered by ASP2010 funds or by the home institute of the lecturers.

The lecturer accommodation expenses paid from ASP2010 funds amounted to $€ 20,302$. Some lecturer accommodation expenses were covered directly by their home institutes in addition to the contributions shown in Table 1 and in Fig. 3. Figure 4 shows the number of accommodation days paid from ASP2010 funds or by the home institutes of the lecturers.

Further details on the usage of the funds, in particular for the students, can be found in Section 5.1.

\subsection{Student Selection}

A total of 65 students participated in ASP2010. Among these students, 50 students, from 17 different African countries, were selected and their transportation, accommodation and meals fully covered; one student was selected from India and also had his transportation, accommodation and meals fully funded. Four students from Canada, Germany, the USA and Switzerland had to 
Table 1: Summary of the ASP2010 budget. In addition to the contributions mentioned in this table, the following institutes covered travel and/or accommodation costs for their lecturers: BNL, FNAL and ICTP paid for travel and accommodation costs for three lecturers (one from each institute); CERN paid travel costs for ten lecturers; PSI and EPFL paid travel costs for two lecturers (one from each institute). The CNRSIN2P3 contribution of $€ 28000$ includes $€ 5000$ travel cost that is reported in the expenses as "Int. Committee Meetings".

\begin{tabular}{|c|c|}
\hline \multicolumn{2}{|l|}{ Incomes (€) } \\
\hline European Contribution & 106,970 \\
\hline U. Santiago de Compostela / Ministry of Foreign Affairs - AECID - Spain & 30,000 \\
\hline CNRS IN2P3 - France & 28,000 \\
\hline Int. Center for Theoretical Physics (ICTP) - Italy & 25,000 \\
\hline CERN (20,000 CHF) - Switzerland & 13,970 \\
\hline CEA - France & 4,000 \\
\hline CNRS Institut des Grilles - France & 3,000 \\
\hline Paul Scherrer Institute (PSI) / Ecole Polytech. Féd. de Lausanne (EPFL) - Switzerland & 3,000 \\
\hline American Contribution - USA & 26,180 \\
\hline Fermi National Accelerator Laboratory (FNAL) / Department of Energy (DOE) $\$ 10,000$ & 7,930 \\
\hline T. Jefferson Nat. Acc. Facility (TJNAF) $\$ 10,000$ & 7,930 \\
\hline Brookhaven Nat. Lab. (BNL) $\$ 5,000$ & 3,970 \\
\hline National Science Foundation (NSF) $\$ 8,000$ & 6,350 \\
\hline South African Contribution & 23,920 \\
\hline Nat. Inst. for Theoretical Physics (NITheP), Stellenbosch R150,000 & 15,600 \\
\hline Nat. Research Foundation (NRF), Pretoria R80,000 & 8,320 \\
\hline \multicolumn{2}{|l|}{$\begin{array}{l}\text { Total Income } 157,070 \\
\end{array}$} \\
\hline \multicolumn{2}{|l|}{ Expenses (€) } \\
\hline Preparation \& General Costs & 6,300 \\
\hline Int. Committee Meetings & 5,000 \\
\hline Secretariat \& Logistics & 1,300 \\
\hline School Running Costs & 43,884 \\
\hline Classroom rental, coffee breaks, catering & 35,896 \\
\hline Internet \& Wifi (U. of Stellenbosch) & 2,080 \\
\hline Transportation (Shuttles, Rental Cars) & 3,063 \\
\hline Insurance for non-SA Students & 1,969 \\
\hline Students Local Expenses & 876 \\
\hline Travel \& Accommodation Costs (without catering) & 92,726 \\
\hline for Students & 54,424 \\
\hline for Lecturers / Tutors & 38,302 \\
\hline Social Events & 6,436 \\
\hline Excursion (Aug. $8^{\text {th }}$ ) & 2,940 \\
\hline School Banquet (Aug. 15 ${ }^{\text {th }}$ ) & 3,496 \\
\hline Communication \& Organisation & 6,656 \\
\hline Videoconferences \& Broadcasts & 4,205 \\
\hline Committee Organising Expenses & 2,136 \\
\hline School Package \&Lectures Material DVD & 315 \\
\hline Contingency remaining & 1,068 \\
\hline Total Expenses 157,070 & \\
\hline
\end{tabular}




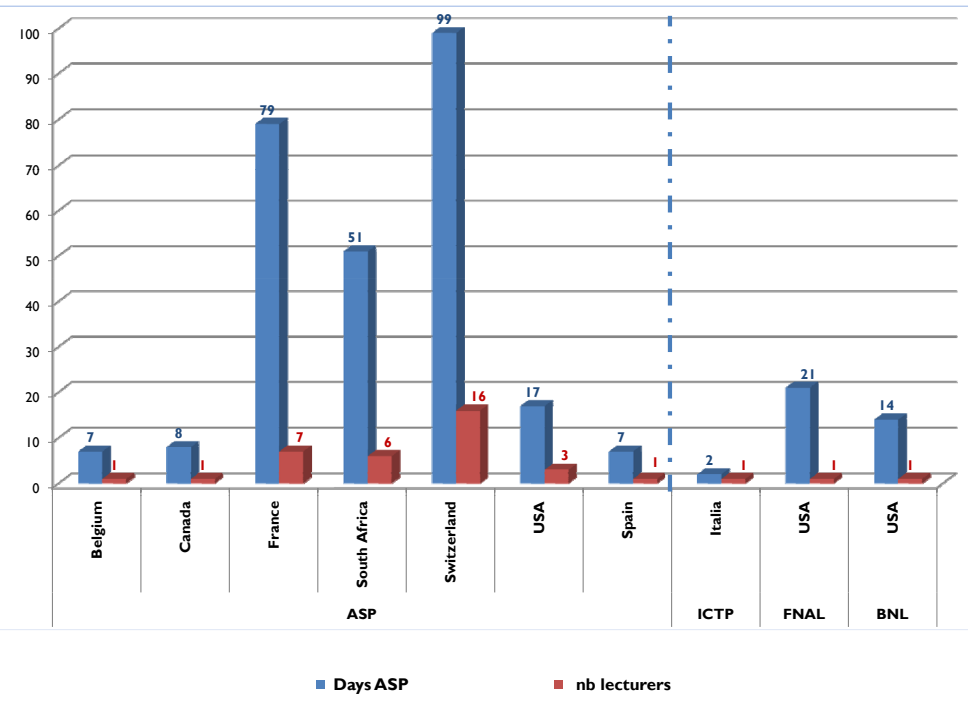

Figure 4: Accommodation days of the lecturers. The blue and red histograms represent respectively the number of accommodation days paid from ASP2010 funds or directly by the institutes, and the corresponding number of lecturers.

pay for their own transportation, while their accommodation and meals were fully covered. The other remaining students were already living in South Africa and most of them were studying at the University of Stellenbosch. Figure 5 shows the distribution of the students, where $16 \%$ of the student were female. The students were selected from more than 150 eligible applicants received before June 2010.

\section{ASP 2010}

In this section, we report on the school itself, i.e., the running of the school during the period of August 1-21, 2010. A few photographs of the first, second and third weeks of the school are shown in the appendix.

\subsection{Logistical Support}

The support provided by the host country in terms of infrastructure is essential to the success of the school. The logistical support offered by the host country is one of the criteria in the selection of the venue as explained in Section 3.1. In this section, we provide some feedback on the logistical support for ASP2010. The ASP2010 was hosted by the NITheP [9] in Stellenbosch. Some experimental courses and demonstrations were also carried out at iThemba LABS [10]. The lecture hall could accommodate all the students, lecturers and organising committee members for plenary sessions, an atmosphere that encouraged questions from the students and invited discussions. The facility also offered different halls and rooms for coffee breaks, lunch and breakout sessions for 


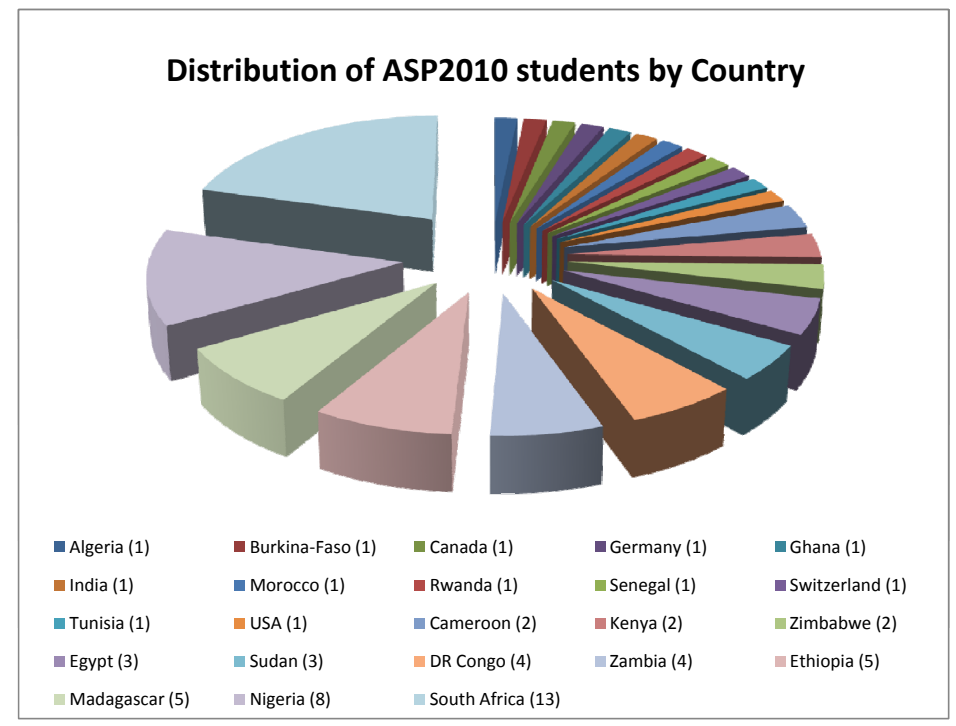

Figure 5: The distribution of the selected ASP2010 students by country.

small group topical discussions. The lectures that required hands-on computing (Grid computing and GEANT4 [11] exercises) and physics experiments where carried out in different departments (that offer the needed equipment) within walking distance of the university, or at iThemba LABS (by pre-arranged group transportation).

\subsection{Lectures}

The details of the lectures, discussion and practical sessions are documented on the ASP2010 website [1] and followed the main topics as outlined in Section 3.2. The theoretical physics (TH) theme was concentrated in the first week of the school. The Experimental Subatomic Physics (EP) theme dealt with what we know about subatomic physics including experiments results and methods. It formed the core of the lecturers in the second week. The Information Technology (IT) theme mainly dealt with practical sessions on Grid computing, spread over the three-week period. The scope of the third week's courses was for the students to learn the basics of particle accelerator technology and applied physics (AT). The scientific disciplines of medical physics, material and biochemical based research using synchrotron radiation and magnetic confinement fusion were discussed.

A complete set of all the lectures and tutorial sessions was written on DVD and were given to each student before leaving ASP2010.

\subsection{Discussion Sessions}

Most academic lectures were followed by discussion sessions in smaller groups typically between ten and twenty students. These groups were formed to best match the broad range of interests 
and academic levels of the students (since the school was open to both graduate and undergraduate students). This allowed the discussion leaders to address more efficiently the needs of the students.

\subsection{Practical Sessions}

To compliment the lectures, practical exercises, tutorials and laboratory sessions were organised, on event generation using PYTHIA [15], on detailed GEANT4 [11] simulation, on data acquisition and on data analysis in ROOT [13], to give the students "hands-on" scientific training. During these practical sessions, the students became acquainted with the use of scintillation detectors and performed measurements of environmental radioactivity.

\section{Follow-up}

In this section, we discuss activities after the school. These include balancing the budget, the feedback from the students and maintaining contact with the students.

\subsection{Balancing the Budget}

The main priority of the budget was to organise and run ASP2010 with a full coverage of the travel, accommodation and living expenses for students coming from sub-Saharan Africa, and also invite students from Northern Africa, and fewer from Europe, the USA and elsewhere to provide a multicultural setting, meant to initiate networking and to share experiences in learning physics and pursuing research in this field.

Figure 6 shows the breakdown of the origin of the funds and their usage. In total we supported

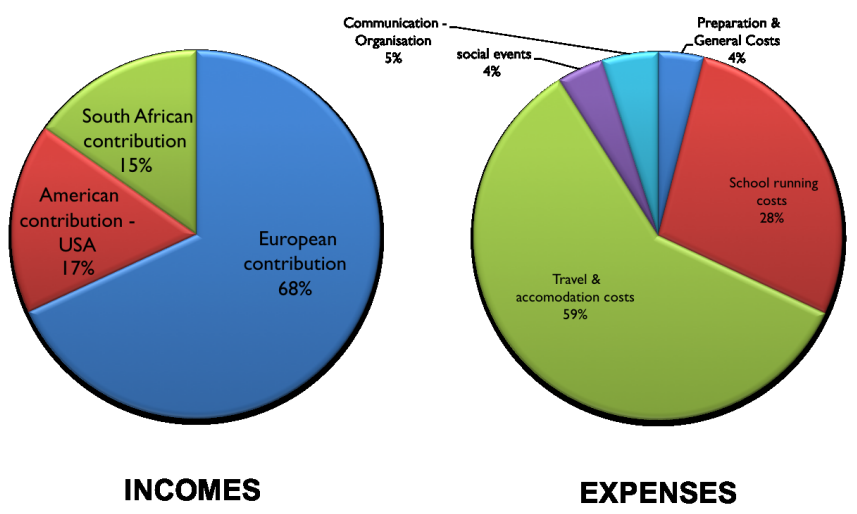

Figure 6: Origin of the funds (left) and of their usage (right).

62 students for the full duration of ASP2010 while 3 local students attended occasionally some selected lectures. The students from Europe, Canada and the USA had their travel paid by their home institutes, the rest relied on ASP2010 funds as shown in Fig. 7.

The estimated budget covered very well all the expenses of the school. There is a minor surplus of about $€ 1,000$. 


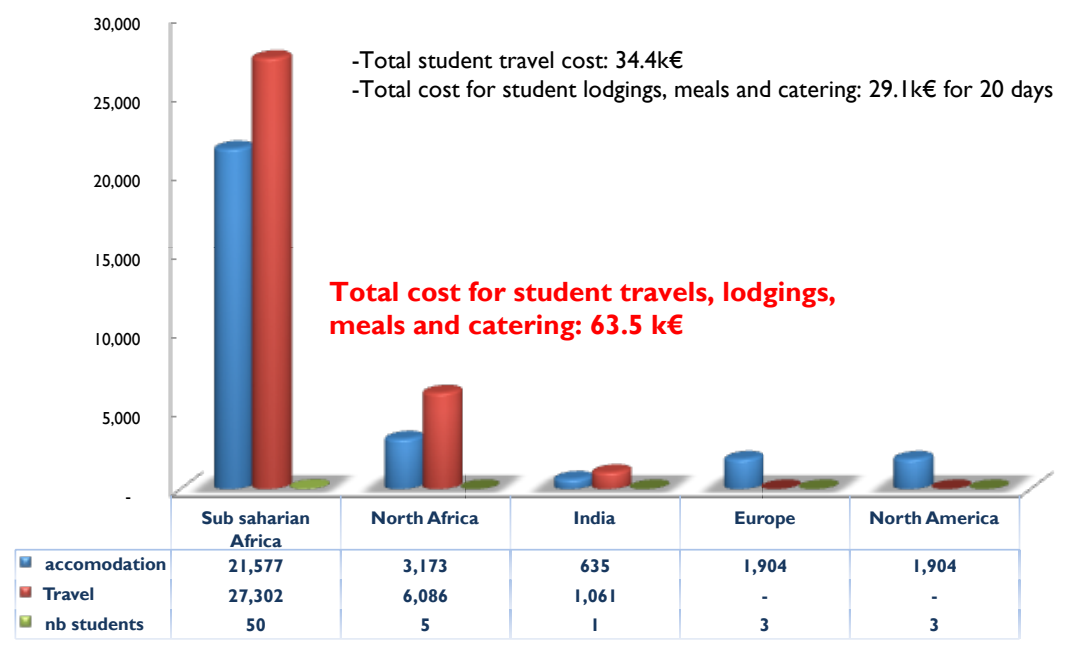

Figure 7: Travel and accommodation (including catering) expenses as a function of the geographical origin of the students. The travel expenses of students from Europe, Canada and the USA were covered by their own institutes.

\subsection{Student Feedback}

By all accounts, the experience was extremely valuable for all the participants. The inspirational enthusiasm of the students at ASP2010 exceeded our expectation and we have received much positive and constructive feedback. Some student feedback has already been included in published press releases [20, 21, 22].

In order to understand the impact of ASP2010 from the students' perspectives two surveys were prepared. The first survey was designed to complete our database and provide us with easy and accessible basic information such as the home institute and degree of each student. The second survey was designed to provide us with feedback about the quality of the school in order to take this into consideration in a future version of the school. A few students also shared their feedback through personal emails to the organisers. The following is a summary of these surveys and feedback.

It seems that most of the students heard about the school through word of mouth, advertisement in their departments or recommendation from their supervisors or colleagues.

By attending ASP2010, most of the students were expecting to learn more about the international high energy physics community, to make contacts through networking with lecturers and to get more information about scholarship and fellowship opportunities specially in North America and Europe. Some were also seeking to get ideas for their future research as well as connecting to other African physicists. Most of these expectations were met to a good extent. These results are summarised in Fig. 8.

Some of the common suggestions to improve the school include increasing the computer lab sessions in order to get more hands-on experience as well as decreasing the variety of physics topics covered in the school. Many students believe that the school was long and there was little social time to interact with lecturers. It seems that there is also room for improvement in providing better 


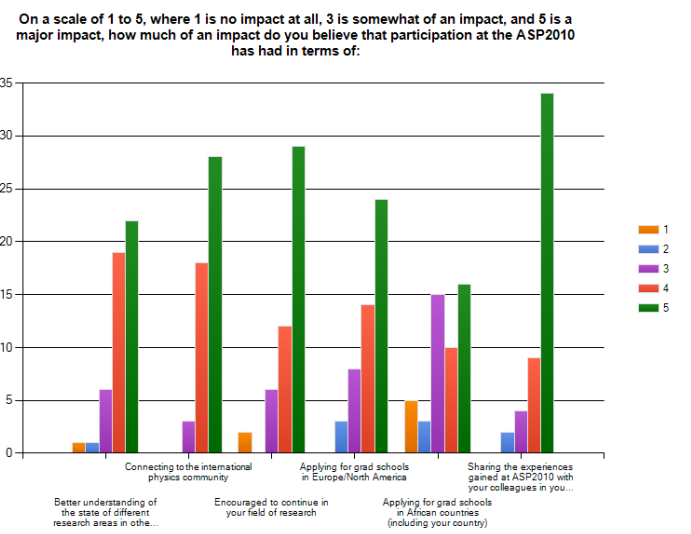

Figure 8: Impact of ASP2010 on the students. On the horizontal axis, the first and the last questions read respectively "Better understanding of the state of different research areas in other African countries, Europe and North America" and "Sharing the experiences gained at ASP2010 with your colleagues in your home institutes/universities".

accommodation for students. Overall, about $37 \%$ of the students are satisfied and 53\% are very satisfied with their experience at ASP2010. 100\% of the students will apply for the school again in future. The results are shown in Fig. 9.
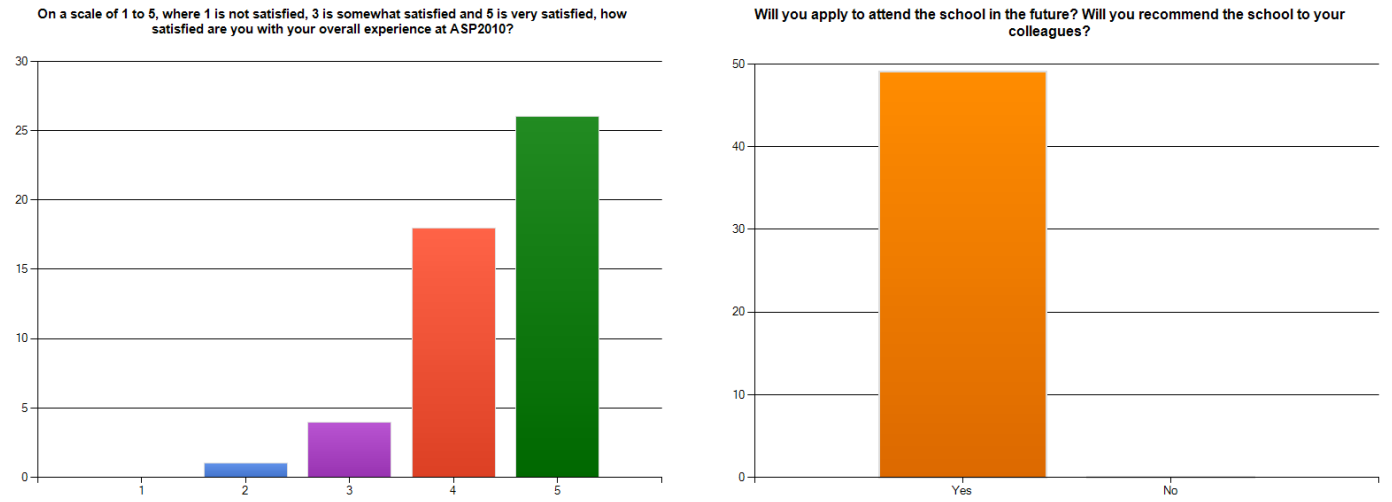

Figure 9: Satisfaction of the students and their willingness to attend the school again.

In response to whether or not the students are interested in scholarship opportunities, more than $90 \%$ of them stated that they would be interested in fellowship opportunities in North America and Europe and about $70 \%$ are also considering opportunities in other African countries. These results are summarised in Fig. 10.

Figures 11 summarises the rating of the lecturers in terms of content of the lectures, clarity and easiness to follow, the speed of the lectures, etc. Overall, the students were very satisfied with the quality of lecturers and sessions. 


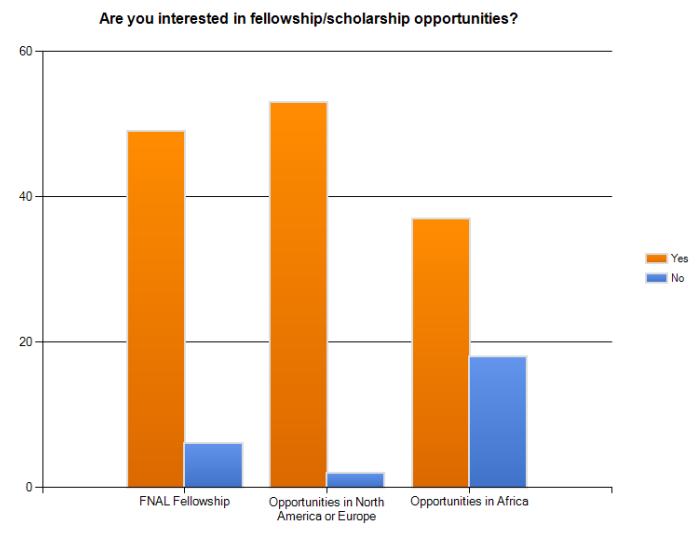

Figure 10: Student interest in fellowships and scholarships.
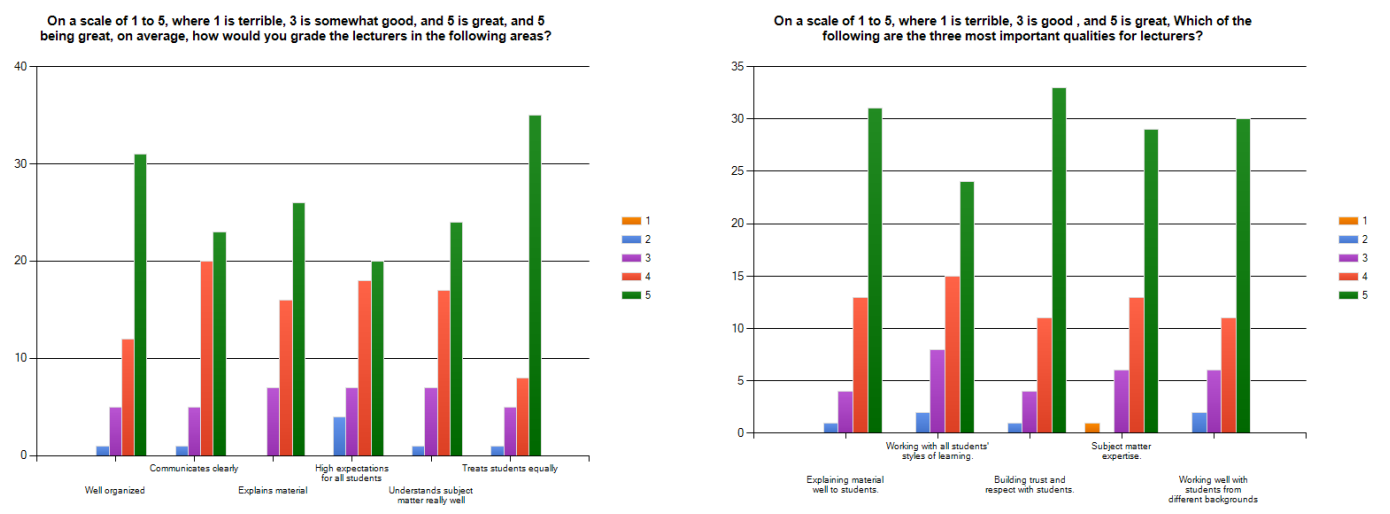

Figure 11: Student feedback on the lectures (left), and Student feedback on lecturers (right).

\subsection{Maintaining Contacts with Students}

It was emphasised throughout the school that the students are main actors in their research careers. However, with a focus on empowering the students to make their own career choices, and in the spirit of increased networking, some career guidance and mentoring was given during ASP2010, by sharing with the students the websites where typically Ph.D. and post doctoral research positions are publicly advertised.

In order to retain contact with the students, a email group list was set-up through CERN [23] and a social networking facebook page [24] was created to share news and information. This has proved to be extremely helpful in communicating interesting physics news to the students and in getting updates on their evolving career paths.

In order to identify a suitable host country and institution for the next ASP school, the contact with the existing students has already proved invaluable, in connecting through them to their universities and institutes to build potential future collaborating partnerships.

Since the ASP2010 school, the South African Department of Science and Technology has 
shown an increased support to collaborating scientists in South African universities and national laboratories, who are working within the research consortium, "SA-CERN", to participate fully in the CERN research experiments of ALICE, ATLAS, ISOLDE [25] and the supporting theoretical physics activities.

\section{Outlook}

The success of the school is due to the financial support from thirteen institutes in the USA, in Europe and in South Africa, to the dedication of the organising committee, to the devotion of the lecturers, and to the interests of the students themselves. Many students in Africa face challenges in terms of the logistical support, the quality of education and the opportunity for higher education abroad. Some of us in the organising committee had faced these challenges ourselves. It is often the case in Africa that even the best students do not have the needed support to succeed or to acquire the necessary skills to be competitive at an international level. It was particularly important for the ASP2010 organising committee to do something, to be part of something where one could help resolve some of the challenges that students from Africa face. It is not to suggest that this particular school has solved all the issues, not at all. However, it is hoped that this school was useful in terms of networking, which in turn will help prepare the students to find practical answers to many issues that they may need to resolve. It is further hoped that this will not be the first and the last school.

Looking at the long term objectives (to help improve high training and education in Africa) that motivated the organisation of ASP2010, the current success, although encouraging, is rather limited in scope. Firstly, the school resources only allowed for 65 students to be accommodated. That was sufficient for the efficient management of the school but it is only a small step in the right direction to making a significant impact. Secondly, the duration the school, although appropriate given the constraints from the budget, students and lecturers, could not allow for a more extended coverage of the topics that were presented. Thirdly, the budget available for the school could not allow a longer duration with more time spent on the details of each topic. Finally, as can be seen in Fig. 5, the participation of students from French speaking African countries could be improved. All these are not a failure of ASP2010 but rather a motivation to work harder towards the original objectives by organising the school again in the future, and in doing so, truly contribute in a significant way to development in Africa, and indeed to the betterment of humankind.

Fermilab is committed to support three of the ASP2010 students up to two years for an equivalent of $\$ 25,000$ per student per year. They will be stationed at Fermilab and receive this additional support towards their post-doctoral studies.

To build up on the success of ASP2010, the organising committee proposes a similar school in 2012, ASP2012, but in a different African country. The committee is already exploring this option and a number of host countries has been suggested. In time, the committee will select the host country and approach the funding institutes of ASP2010 for support for the ASP2012, and the process of identifying students and lecturers will be carried out. Early involvement of the local host in the organisation process is essential to the success of the school. 


\section{Conclusions}

For the past few years, a group of local and international organising committee members have worked very hard to prepare for the first biennial school of fundamental physics and its application in Africa. Finally, the efforts of the organising committee and all the supporting institutes and concerned individuals paid off and the school took place in Stellenbosch, South Africa on August 1-21, 2010. A total of about 65 students from all over Africa (including a few from Europe, America, one from India) attended the school. There was also the participation of high profile international and local lecturers who prepared and presented the materials taught during the school. They included theoretical and experimental particle physics, particle accelerators and practical applications. The last day of the school was devoted to outreach where a review of the main objectives of the school was presented and discussed. There were also presentations and discussions on the enhancement of science, technology and innovation in South Africa and in Africa in general.

Friendly atmosphere throughout the school encouraged direct contacts between the students and the lecturers and to hear the student's concerns about the possibility of pursuing higher education. The students established contacts and network with the lecturers and with other students; we expect these connections to be useful to the students and to be maintained far the beyond the school itself. Feedback from students and lecturers suggests that it was a very successful and well received school, and that there is a demand for the school to be organised again within the next two years.

\section{Acknowledgements}

We would like to thank the universities, research institutes and government agencies that provided the funding for ASP2010, namely, the Spanish AECID Ministry of Foreign Affairs, ICTP, IN2P3 (France), l'Institut des Grilles (France), le Commissariat à l'énergie atomique (France), Ecole Polytechnique Fédérale de Lausanne (Switzerland), Paul Scherrer Institute (Switzerland), NITheP (South Africa), National Research Foundation (South Africa), Fermilab (USA), Department of Energy (USA), Brookhaven National Laboratory (USA), Jefferson Lab (USA), National Science Foundation (USA), CERN and the International Union of Pure and Applied Physics (IUPAP).

\section{References}

[1] S. Muanza et al., African School of Fundamental Physics and its Applications, Available from http://africanschoolofphysics.web.cern.ch, 2010.

[2] The LHC Project Collaboration, The Large Hadron Collider, Available from http://lhc.web.cern.ch/lhc/.

[3] CDF and D0 Collaboration, T. Aaltonen et al., Combination of Tevatron searches for the standard model Higgs boson in the W+W- decay mode, Phys. Rev. Lett. 104 (2010) 061802, arXiv:1001.4162 [hep-ex].

[4] The ATLAS Collaboration, Expected Performance of the ATLAS Experiment - Detector, Trigger and Physics, Http://public.web.cern.ch/public/en/lhc/atlas-en.html, 2008. arXiv:0901.0512 [hep-ex] (2008) . 
[5] The CMS Collaboration, CMS Physics Technical Design Report, Volume II: Physics Performance, Http://public.web.cern.ch/public/en/lhc/cms-en.html, 2007. J. Phys. G: Nucl. Part. Phys. 36 (2007) .

[6] The ALICE Collaboration, A Large Ion Collider Experiment, Available from http://public.web.cern.ch/public/en/lhc/alice-en.html.

[7] The LHCb Collaboration, Large Hadron Collider Beauty, Available from http://public.web.cern.ch/public/en/lhc/lhcb-en.html.

[8] The Abdus Salam International Centre for Theoretical Physics, Http://www.ictp.it/.

[9] National Institute of Theoretical Physics, Http://www.nithep.ac.za/.

[10] iThemba - Laboratory for Accelerator Based Sciences, Http://www.tlabs.ac.za/.

[11] S. Agostinelli et al., GEANT 4 a simulation toolkit, NIM A 506 (2003) 250-303.

[12] CERN Accelerator Complex Control Centre, Http://public.web.cern.ch/public/en/spotlight/spotlightccc-en.html.

[13] R. Brun et al., ROOT Reference Guide, Available from http://root.cern.ch.

[14] The ITER Project, Http://www.iter.org/.

[15] T. Sjostrand et al., PYTHIA 6.4 physics and manual, JHEP 05 (2006) 026.

[16] The Lanzerac Winery, Http://www.lanzerac.co.za/index.php?page=winery.

[17] African School of Physics: Photos Flicker, Http://www.flickr.com/photos/africanschoolofphysics/.

[18] Saturday Aug 21st - Forum Day: A Gateway to Innovation, Https://africanschoolofphysics.web.cern.ch/africanschoolofphysics/forum.html/.

[19] MTN Sciencentre, 407 Canal Walk, Cape Town, Http://www.mtnsciencentre.org.za/.

[20] First African School of Physics brings cutting-edge physics and technology to sub-Saharan Africa, $\mathrm{Http}: / / \mathrm{www}$.interactions.org/cms/?pid=1029800.

[21] The rainbow school of physics, Http://cdsweb.cern.ch/journal/cernbulletin/2010/35/news

[22] Into Africa -âĂŞ a school in fundamental physics, Http://cerncourier.com/cws/article/cern/44114.

[23] Email List maintained by ASP2010 organizing committee through CERN, Asp-students-network@cern.ch.

[24] African-School-of-Physics: Facebook Page, Http://www.facebook.com/.

[25] The ISOLDE Collaboration, Isotope Separator On Line, Available from http://isolde.web.cern.ch/isolde/. 